\title{
Ginseng/Lingzhi Mushroom/Cordyceps sinensis/Rose Oral Liquid
}

National Cancer Institute

\section{Source}

National Cancer Institute. Ginseng/Lingzhi Mushroom/Cordyceps sinensis/Rose Oral

Liquid. NCI Thesaurus. Code C156397.

An orally available supplement containing ginseng, lingzhi mushroom, Cordyceps sinensis, and rose with potential immunostimulating activities. Upon oral administration, ginseng/lingzhi mushroom/Cordyceps sinensis/rose oral liquid may, through a not yet elucidated mechanism, enhance immune responses and relieve fatigue. 